\title{
ON RELATIONS BETWEEN BROWN-PETERSON COHOMOLOGY AND THE ORDINARY MOD $p$ COHOMOLOGY THEORY
}

\author{
Dedicated to Professor Nobuo Shimada on his 60-th birthday \\ By NoBUaki Yagita
}

\section{Introduction.}

Let $H^{*}\left(X ; Z_{p}\right)$ be the ordinary $\bmod p$ cohomology for odd prime and let $B P^{*}(X)$ be the Brown-Peterson cohomology theory with $B P^{*}=Z_{(p)}\left[v_{1}, v_{2}, \cdots\right]$. The spectrum $V(n)$ is defined by $H^{*}\left(V(n) ; Z_{p}\right) \cong \wedge\left[Q_{0}, \cdots, Q_{n}\right][8]$ where $Q_{\imath}$ is the Milnor operation and $V(n)$ is also defined by $B P^{*}(V(n)) \cong B P^{*} /\left(p, v_{1}, \cdots, v_{n}\right)$ [6]. To consider the equivalence of the above two definitions was the begining of this paper.

We note a relation between the $Q_{i}$-action and $v_{i}$-torsion, which is an immediate consequence from the Sullivan's bordism theory of manifolds with singularities.

LEMMA 2.1. Let $x_{j} \in B P^{*}(X)$ and $\sum v_{j} x_{j}=0 \bmod I_{\infty}^{2}$ where $I_{\infty}=\left(p, v_{1}, \cdots\right)$. Then there is $y \in H^{*}\left(X ; Z_{p}\right)$ such that $Q_{j}(y)=i\left(x_{j}\right)$ where $\imath$ is the inclusion map (Thom map) $\imath: B P \rightarrow H Z_{p}$.

The Brown-Peterson cohomology is studied by many authors, especially the Adams spectral sequence for $B P^{*}\left(S^{N}\right)$ is well researched. However, known examples of non free $B P^{*}$-module $B P^{*}(X)$ are not so many. Using above lemma, we consider the way to calculate $B P^{*}(X)$ when the Steenrod algebra structure of $H^{*}\left(X ; Z_{p}\right)$ is known, and we give examples of the $B P^{*}$-module $B P^{*}(X)$.

In section 1 using Sullivan's original definition of the bordism theory with (cone type) singularities, we treat the Quillen's geometric approach to the cobordism theory. In $\S 2$ main lemmas are shown. We recall some important facts about the Atiyah-Hirzebruch spectral sequence and we define an invariant which is convenient to use. Some examples are discussed in $\S 3$. The spectrum $V(n)$ and Lens spaces are first treated. We next study about finite $H$-spaces and Eilenberg-Maclane space $K(Z, 3)$, in particular, $B P^{*}$-module structures of even dimensional indecomposed elements are discussed.

The author thanks to W.S. Wilson who suggested the proof of Theorem 3.4.2.

Received October 3, 1983 


\section{$\S 1$. Cobordism theory with singularities.}

We first recall the definition of manifolds with singularities due to Baas [1]. Let $S_{n}=\left(P_{1}, \cdots, P_{n}\right)$ be a sequence of closed manifolds. We say $Z$ is an $S_{n^{-}}$ manifold if its boundary is decomposed as products of $P_{\imath}$, namely, there are manifolds $\partial_{\imath} Z, \partial_{\left(\imath_{1}, \cdots, \imath_{s}\right)} Z, Z\left(i_{1}, \cdots, i_{s}\right)$, for $\left(i_{1}, \cdots, i_{s}\right) \subset(1, \cdots, n)$, and natural isomorphisms

$$
\begin{aligned}
& \partial Z \cong \cup \partial_{\imath} Z \\
& \partial_{\imath_{1}} Z \cap \cdots \cap \partial_{\imath_{s}} Z=\partial_{\left(\imath_{1}, \cdots, \imath_{s}\right)} Z \cong Z\left(\imath_{1}, \cdots, \imath_{s}\right) \times P_{\imath_{1}} \times \cdots \times P_{\imath_{s}} .
\end{aligned}
$$

We next define an $\hat{S}_{n}$-manifold $\left(S_{n}\right.$-manifold with cones) by the quotient space of $Z$ collapsing the $P_{i}$-factors, i.e.,

$$
\begin{gathered}
\hat{Z}=Z /(w, a) \sim(w, b) \text { where }(w, a),(w, b) \in \partial_{\left(i_{1}, \cdots, \imath_{s}\right)} Z \\
\text { and } w \in Z\left(i_{1}, \cdots, \imath_{s}\right), a, b \in P_{\imath_{1}} \times \cdots \times P_{\imath_{s}} .
\end{gathered}
$$

Sullivan originally defined an $\hat{S}_{n}$-manifold as the manifold such that each point of its boundaries has a neighbourhood factored cone $\left(P_{\imath_{1}} * \cdots * P_{\imath_{s}}\right)$. We show these two definition are equivalent.

Consider the tubular neighbourhoods of $\partial_{(1, \ldots, s)} Z$,

(Nei. in $\partial Z) \cong Z(1, \cdots, s) \times P_{1} \times \cdots \times P_{s} \times \Delta_{s-1}$,

(Nei. in $Z) \cong Z(1, \cdots, s) \times P_{1} \times \cdots \times P_{s} \times \Delta_{s}$

where we identify $\partial_{(1, \cdots, s)} Z=Z(1, \cdots, s) \times P_{1} \times \cdots \times P_{s} \times\left(\right.$ center of $\left.\Delta_{s-1}\right)$ and $\Delta_{s-1}$ incluses the $s+1$-th face of $\Delta_{s}$. Take the boundary of Nei. in $Z$ (the link complex)

$$
L k \cong Z(1, \cdots, s) \times P_{1} \times \cdots \times P_{s} \times\left(\dot{\Delta}_{s}-\dot{\Delta}_{s-1}\right) .
$$

Since $\left(\dot{\Delta}_{s}-\stackrel{\Delta}{s-1}\right) \cong \Delta_{s-1}$, if we take the quotient (2), then

$$
\begin{aligned}
\widehat{L k} & \cong Z(1, \cdots, s) \times P_{1} \times \cdots \times P_{s} \times \Delta_{s-1} /\left(w, \cdots p_{i}, \cdots, \sigma\right) \sim\left(w, \cdots p_{i}^{\prime}, \cdots, \sigma\right), \sigma \in \dot{\Delta}_{s-1} \\
& \cong Z(1, \cdots, s) \times P_{1} * \cdots * P_{s} .
\end{aligned}
$$

Since boundary $\partial_{(1, \ldots, s)} Z$ collapses to $Z(1, \cdots, s)$, (Nei. in $Z$ ) collapses to

$$
\text { (Nei. } Z) \cong Z(1, \cdots, s) \times\left(\text { cone } P_{1} * \cdots * P_{s}\right) \text {. }
$$

Hence the definition (2) is the Sullivan one. Moreover we note $\hat{Z}$ is also defined by

$$
\hat{Z}=\hat{Z} \cup \Sigma J(i) \times Z(i) \cup \Sigma j(i, j) \times Z(\imath, j) \cup \cdots \cup J(\imath, \cdots, n) \times Z(1, \cdots, n)
$$

where $J(1, \cdots, s)=$ cone $\left(P_{1} * \cdots * P_{s}\right)$

$$
=\text { cone }\left(J(2, \cdots, s) \times P_{1} \cup \cdots \cup J(\cdots, \hat{t}, \cdots) \times P_{t} \cup \cdots \cup J(1, \cdots, s-1) \times P_{s}\right)
$$

and cone $P=P \times I / P \times\{1\}$ and $\partial_{(1, \ldots, s)} Z=\{0\} \times Z(1, \cdots, s)$.

Hereafter let denote $\partial_{(i, \ldots, s)} \hat{Z}=\hat{Z}(1, \cdots, s)$. 
DEFINITION 1.2. Let $X$ be an (open or closed) manifold and $\hat{Z}$ be an $\hat{S}_{n^{-}}$ manifold. A map $f: Z \rightarrow X$ is a complex oriented of dimension $q$ if $f$ is factored such that

$$
f: \hat{Z} \stackrel{i}{\longrightarrow} X \times R^{N} \stackrel{p}{\longrightarrow} X
$$

(1) $i$ is an embedding with normal bundles $\nu_{i_{1} \cdots \nu_{s}}$ having compatible stable complex structure on each $\left.\left(\partial_{i_{1} \cdots i_{s}}\right) \hat{Z}-\partial\left(\partial_{\left(i_{1} \cdots i_{s}\right)} Z\right)\right)$,

(2) $p$ is a projection,

(3) if $z \in\left(\partial_{\left(i_{1}, \cdots, i_{s}\right)} \hat{Z}-\partial\left(\partial_{\left(i_{1}, \cdots, i_{s}\right)} Z\right)\right.$, then $(\operatorname{dim} Z$ at $z)-(\operatorname{dim} X$ at $f(z))=q-\left(\operatorname{dim} P_{\imath_{1}}+\cdots+\operatorname{dim} P_{\imath_{s}}+s\right)$.

DeFinition 1.3. Let $f: \hat{Z} \rightarrow X$ be a complex oriented map and $g: Y \rightarrow X$ be a map. Define the modified pull back $Y \times_{X}^{\prime} Z \rightarrow Y$ as follows.

For ease of arguments, assume $n=1$, i.e., the $S_{1}$-case. Let $\hat{Z}=Z \cup$ cone $P_{1} \times Z(1)$. Take $g^{\prime} \times f^{\prime}(1)$ transversal to the diagonal $\Delta \subset X \times X$. Then $\left(g^{\prime} \times f^{\prime}(1)\right)^{-1} \Delta$ $=Y \times{ }_{X} Z(1)$ is a manifold and $\left(g^{\prime} \times f^{\prime}(1) \cdot \text { porj }\right)^{-1} \Delta=Y \times{ }_{X} Z(1) \times \operatorname{cone} P_{1}$ where proj: $Z(1) \times$ cone $P_{1} \rightarrow Z(1)$ is the projection. Let $f^{\prime \prime}: Z \cup \partial Z(1) \times I \rightarrow X$ be a map so that $f^{\prime \prime}=f$ on $Z$ and $f^{\prime \prime}$ is the homotopy between $f$ and $f^{\prime}$ on $Z(1) \times I$. Taking $g^{\prime} \times f^{\prime \prime \prime}$ for $g^{\prime} \times f^{\prime \prime}$ transversal to $\Delta$, we can define

$$
\left(g^{\prime} \times\left(f^{\prime \prime \prime} \cup f^{\prime}(1) \cdot \operatorname{proj}\right)\right)^{-1}=Y \times_{X}^{\prime} Z .
$$

When $n>1$, we can also define the modified pull back by descending induction on sequences $\left(i_{1}, \cdots, i_{s}\right)$ in $(3)$.

Definition 1.4. Let $f_{\imath}: Z_{\imath} \rightarrow X, \imath=1,0$ be complex oriented maps. Then they are cobordant if there is a proper complex oriented map $b: W \rightarrow X \times R$ such that $\varepsilon_{\imath}: X \rightarrow X \times R, \varepsilon_{i}(x)=(x, i)$ is transversal to $b$, and the pull back of $\varepsilon_{\imath}$ is isomorphic to $f_{\imath}$.

THEOREM 1.5. For a manifold $X$, the set of cobordism classes of proper complex oriented map of dimension $-q$ is $M U\left(S_{n}\right)^{q}(X)$, Here $M U\left(S_{n}\right)^{*}(X)$ is the cobordism theory with singularities and without cone due to Baas [1], [9].

Definition 1.6. (Gysin homomorphism) A proper complex oriented map $g: \hat{X} \rightarrow Y$ of dimension $d$ induces a map

$$
g_{*}: M U\left(S_{n}\right) *(\hat{X}) \longrightarrow M U\left(S_{n}\right)^{*-d}(Y)
$$

which sends $f: \hat{Z} \rightarrow \hat{X}$ into $g f: \hat{Z} \rightarrow Y$.

DeFINITION 1.7. (Contravariant map) Let $g: Y \rightarrow X$ be a map of manifolds, and let $f: \hat{Z} \rightarrow X$ be a proper complex oriented map. Then $g$ induces a map

$$
g^{*}: M U\left(S_{n}\right) *(X) \longrightarrow M U\left(S_{n}\right) *(Y)
$$

which sends $f: \hat{Z} \rightarrow X$ into the modified pull back $Y \times_{X}^{\prime} \hat{Z} \rightarrow Y$. 
THEOREM 1.8. (Sullivan's exact sequence) There is an MU*-module exact sequence

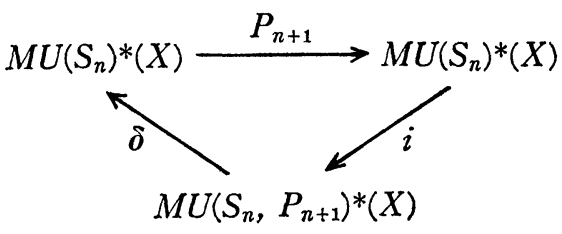

where $\imath$ is the natural inclusion map and $\delta(\hat{A}, f)=\left(\partial_{n+1} \hat{A}, f\right)$.

COROLLARY 1.9. If $S_{n}$ is a regular sequence in $M U^{*}$, then $M U\left(S_{n}\right) *\left(S^{0}\right)$ $\cong M U^{*} /\left(S_{n}\right)$.

In particular $M U\left(x_{\imath} \mid i \neq p^{\jmath}-1\right)^{*}(X)_{(p)} \cong B P^{*}(X)$ and $M U\left(p, x_{1}, \cdots\right)^{*}(X) \cong H^{*}$ $\left(X ; Z_{p}\right)$. Identifyning $x_{p^{2-1}}=v_{i}$, we denote $M U\left(S_{n} x_{\imath}, \cdots \mid i \neq p^{2}-1\right)^{*}(X)_{(p)}$ by $B P\left(S_{n}\right)^{*}(X)$. Recall the notations $B P\left(p, v_{1}, \cdots, v_{n-1}\right)=P(n), B P\left(p, \cdots, v_{n-1}\right.$, $\left.v_{n+1}, \cdots\right)=k(n)$ and $v_{n}^{-1} k(n)=K(n)$.

Define an operation $Q_{P_{i}}$ by $Q_{P_{i}}([\hat{A}, f])=\left[\partial_{i} \hat{A}, f \mid \partial_{i} \hat{A}\right]=[\hat{A}(i), f(i)]$. Then it is easily seen $Q_{P_{i}} Q_{P_{j}}=-Q_{P_{j}} Q_{P_{2}}$. Hereafter we fix the generators $v_{n}$ such that the Chern number $c \Delta_{p_{n-1}}\left(v_{n}\right)=p$ mod $p^{2}$, namely, the Milnor manifolds.

THEOREM 1.10. In $H^{*}\left(X ; Z_{p}\right) \cong B P\left(p, v_{1}, \cdots\right)^{*}(X)$, the operation $Q_{v_{i}}$ is the Milnor operation $Q_{\imath},\left(Q_{0}=\right.$ the Bockstein operation and $\left.Q_{\imath}=\mathscr{P}^{p^{\imath-1}} Q_{\imath-1}-Q_{\imath-1} \mathscr{Q}^{p^{\imath-1}}\right)[9]$.

The cohomology operations in $M U^{*}(-)$ are $M U^{*}$-generated by the LandweberNovikov operation $s_{\alpha}$. The operation $s_{\alpha}$ is defined also in $M U\left(I_{n}\right)^{*}(-), I_{n}=$ $\left(p, v_{1}, \cdots, v_{n-1}\right)[9]$. We here define it from geometric viewpoints, as follows.

Given $[\hat{A}, f] \in M U\left(I_{n}\right)^{*}(X)$, we will define $s_{\alpha}[\hat{A}, f]$. First suppose $\partial \hat{A}=$ $\partial_{i} \hat{A}$, i.e., $\partial \hat{A}=A(i) \times v_{i}$. Let $\tau_{\partial A}: \partial A \rightarrow B U$ be the map which represents the tangent bundle of $\partial A$. Since

$$
\tau_{\partial A}^{*} \cong\left(\tau_{A(i)} \times \tau_{v_{\imath}}\right)^{*} \text { and } \tau_{\partial A}^{*}\left(c_{\alpha}\right)=\sum_{\alpha=\alpha^{\prime}+\alpha^{*}} \tau_{A(i)}^{*} c_{\alpha^{\prime}} \tau_{v_{i}}^{*} c_{\alpha^{*}},
$$

the definition of $s_{\alpha}$ in $M U^{*}(-)$ theory follows

$$
s_{\alpha}[\partial A, \partial f]=\sum_{\alpha=\alpha^{\prime}+\alpha^{\prime \prime}} s_{\alpha^{\prime}} A(i) \cdot s_{\alpha^{\prime \prime}}\left(v_{\imath}\right) .
$$

Here $s_{\alpha^{*}}\left(v_{\imath}\right) \in I_{\imath}=\left(p, \cdots, v_{i-1}\right)$ and we can write

$$
s_{\alpha}[\partial A, \partial f]=\sum_{\alpha=\alpha^{\prime}+\alpha^{*}} s_{\alpha^{\prime}} A(i) \cdot \sum_{j<i} b_{\alpha^{\prime \prime}, v_{j}}(1) .
$$

Let $[M, g] \in M U^{*}(A)$ be a manifold which represents $\tau_{A}^{*}\left(c_{\alpha}\right)$. Then there is a manifold $W$ so that

$$
\partial W=\partial M \vee(\text { right hand side of (1)). }
$$

Therefore we can define $s_{\alpha}[A, f]$ by 


$$
g f: M \bigcup_{\partial M} W \cup \sum_{\alpha^{\prime}, j}\left(s_{\alpha^{\prime}}(A(i)) \times b_{\alpha^{*}, \jmath}\right) \times \text { conev }_{\jmath} \longrightarrow A \cup A(i) \times \text { conev }_{\jmath} \longrightarrow X .
$$

The fact that if $b_{\alpha^{\prime} j}$ is also in $I_{\imath-1}$ then $b_{\alpha^{*}, \jmath}=0$ in $M U\left(I_{n}\right)^{*}$ implies that $s_{\alpha}[A, f]$ is welldefined.

The case $\partial A=\cup \partial_{i} A$ is also proved by descending induction on sequences $\left(i_{1}, \cdots, i_{s}\right)$ in $(3)$.

The cohomology operations in $M U\left(I_{n}\right)$-theory is known

$$
M U\left(I_{n}\right)_{*}\left(M U\left(I_{n}\right)\right) \cong M U^{*} / I_{n} \otimes M U^{*}(M U) \otimes \Lambda\left[Q_{p}, \cdots, Q_{v_{n-1}}\right] .
$$

Therefore we can minic the arguments in Quillen's paper [5]. In particular we can prove

Theorem 1.11. Let $X$ be a finite complex. Then $M U\left(I_{n}\right)^{*}(X)\left(\right.$ resp. $\left.P(n)^{*}(X)\right)$ is generated as a $M U\left(I_{n}\right)^{*}$-module (resp. $P(n)^{*}$-module) by elements of non negative degree.

\section{§2. Main lemmas.} tion $Q_{\imath}$.

In the previous section we noted the geometric mean of the Milnor opera-

LEMMA 2.1. Let $x_{j} \in B P^{*}(X)$ and $\Sigma_{v_{j}} x_{\jmath}=0 \bmod I_{\infty}^{2}$ where $I_{\infty}=\left(p, v_{1}, \cdots\right)$. Then there is $y \in H^{*}\left(X ; Z_{p}\right)$ such that $Q, y=i\left(x_{j}\right)$ where $i$ is the natural inclusion map $i: B P \rightarrow H Z_{p}$.

Proof. Think of $x_{\jmath}$ as an singular manifold. Since $\Sigma v_{\jmath} x_{\jmath}=0 \bmod I_{\infty}^{2}$, there is a manifold $y^{\prime}$ whose boundary is

$$
\partial y^{\prime}=\vee v_{j}\left(x, \vee w_{\jmath}\right) \text { where } w_{\jmath}=0 \bmod I_{\infty} .
$$

Let $y=\hat{y}^{\prime}$ be the $I_{\infty}$-manifold constructed from $y^{\prime}$ attaching cones

$$
\hat{y}^{\prime}=y^{\prime} \bigcup_{\partial y^{\prime}}\left(\operatorname{conev}_{\jmath}\right) \times\left(x, \vee w_{\jmath}\right) \text { and } \partial_{\jmath} \hat{y}^{\prime}=\left(x, \vee w_{j}\right) .
$$

Think of $H^{*}\left(X ; Z_{p}\right)$ as $B P\left(I_{\infty}\right) *(X)$ and we have

$$
i^{\prime} \partial_{\jmath} \hat{y}^{\prime}=Q, \hat{y}^{\prime}=i\left(x, \vee w_{\jmath}\right)=i\left(x_{j}\right),
$$

where $i^{\prime}: B P\left(\cdots, \hat{v}_{\jmath}, \cdots\right)^{*}(X)=k(n)^{*}(X) \rightarrow H^{*}\left(X ; Z_{p}\right)$.

q.e.d.

We next recall the Atiyah-Hirzebruch spectral sequence. Given multiplicative spectrum $A$, let denote by ${ }_{A} E_{r}^{*, *}(X)$ the Atiyah-Hirzebruch spectral sequence which converges to $A^{*}(X)$,

$$
{ }_{A} E_{2}^{*, *} \cong H^{*}\left(X ; A^{*}\right) \Rightarrow A^{*}(X) .
$$

The following lemma is well known [9], 
LEMMA 2.2. The first non zero differentral of the spectral sequence ${ }_{P(n)} E_{r}^{*, *}$ (and ${ }_{k(n)} E_{r}^{*, *}$ ) is $d_{2 p^{n-1}}=v_{n} Q_{n}$.

COROLlARY 2.3. In the spectral sequence ${ }_{P(1)} E_{r}^{*, *}={ }_{B P(-, Z)} E_{r}^{*, *}$, if $d_{r} x=0$ for $r<2 p^{n}-1$ then $d_{2 p n-1}(x)=v_{n} Q_{n}(x) \bmod \left(p, \cdots, v_{n-1}\right)$.

Proof. The natural inclusion map $\imath: P(1) \rightarrow P(n)$ and Lemma 2.2 follow the corollary.

q.e.d.

Recall that an ideal $I$ in $B P^{*}$ is invariant if $\theta(I) \subset I$ for all operations $\theta \in$ $B P^{*}(B P)$.

LEMMA 2.4. Let $x_{1}, \cdots, x_{s} \in_{P(1)} E_{r}^{t, 0}$. Then the $\bmod$ annihilator $A M\left(x_{1}\right)=$ $\left\{a \in P(1)^{*} \mid a x_{1}=0 \bmod \left(x_{2}, \cdots, x_{s}\right)\right.$ in $E_{r}^{t, *\}}$ is invarıant.

Proof. This $A M\left(x_{1}\right)$ is indeed the $\bmod \left(x_{2}, \cdots, x_{s}\right)$ annihilator ideal in $P(1)^{*}$ $\left(X^{t+1} / X^{t-r-2}\right)$. Hence this is an invariant ideal. q. e.d.

We now consider relations between the Atiyah-Hirzebruch spectral sequence and the Sullivan exact sequence.

LEMma 2.5. Let $w x=0$ in $P(1)^{*}(X)$ for $0 \neq w \in P(1)^{*}$, and let $i(x)=x^{\prime} \neq 0$ in $H^{*}\left(X ; Z_{p}\right)$. From the Sullivan exact sequence, there is $y$ in $B P(p, w)^{*}(X)$ such that $\delta y=x$. Then $d_{r} y^{\prime}=\lambda w x^{\prime}$ in ${ }_{P(1)} E_{r}^{*, *}$ where $0 \neq \lambda \in Z_{p}, i(y)=y^{\prime}$ and $r=|w|+1$.

Proof. Since $P(1)^{*}(X)=B P^{*}\left(X ; Z_{p}\right) \cong B P^{*}\left(X \wedge S^{0} \cup_{p} e^{1}\right)$, we consider this lemma in ${ }_{B P} E_{r}\left(X \wedge S^{0} \cup_{p} e^{1}\right)$. Take the normal cells decomposition of $X \wedge S^{0} \cup_{p} e^{1}$, i.e.,

$$
\begin{aligned}
& *=Y_{0} \subset \cdots \subset Y_{\infty} \cong X \wedge S^{0} \cup_{p} e^{1}, \\
& Y_{n}=Y_{n-1} \cup_{f} \operatorname{cone}\left(\bigvee_{k} S^{n} \cup_{p} e^{1}\right), \quad k=\operatorname{dim}_{Z_{p}}\left(H_{n}\left(Y_{\infty} ; Z\right)\right) .
\end{aligned}
$$

Put $Y\left(n-x^{\prime}\right)=Y_{n-1} / Y_{n-r-2}$-cone $\left(S^{n-2} \cup_{p} e^{n-1}\right)_{x^{\prime}}$ where $n=\left|x^{\prime}\right|$ and cone $\left(S^{n-2} \cup_{p} e^{n-1}\right)_{x^{\prime}}$ is the cone of the Moore space which represents $x^{\prime}$ in $H^{*}\left(Y_{\infty} ; Z\right)$. Since $\delta y=x^{\prime}=0$ in $B P^{*}\left(Y\left(n-x^{\prime}\right)\right)$ where $y \in B P(w)^{*}\left(Y\left(n-x^{\prime}\right)\right), y$ is also in $B P^{*}\left(Y\left(n-x^{\prime}\right)\right)$ and $y^{\prime}$ is a permanent cycle in ${ }_{B P} E^{* * *}\left(Y\left(n-x^{\prime}\right)\right)$, i.e., $d_{r} y^{\prime}=0$.

On the other hand put

$$
Y\left(n-1+x^{\prime}\right)=Y_{n-2} / Y_{n-r-2} \cup \text { cone }\left(S^{n-2} \cup_{p} e^{n-1}\right)_{x^{\prime}} .
$$

Then $\delta y=x \neq 0$ and $y^{\prime}$ is not a permanent cycle in ${ }_{B P} E^{*} *\left(Y\left(n-1+x^{\prime}\right)\right)$. Hence $d_{r} y^{\prime}=\lambda w x^{\prime}$.

Therefore $d_{r} y^{\prime}=\lambda w x^{\prime}$ in ${ }_{B P} E^{*, *}\left(Y_{n-1} / Y_{n-r-2}\right)$. By the construction of the spectral sequence we have the lemma.

q.e.d.

The following corollary is an analogous result of Lemma 2.1.

COROLLARY 2.6. Let $\left(w_{1}, \cdots, w_{s}\right)=J_{s},\left|w_{\imath}\right|<\left|w_{\imath+1}\right|$ be a regular sequence in $P(1)^{*}$. Let $b_{j} \in P(1)^{*}(X)$ and $0 \neq i\left(b_{\jmath}\right)$ in $H^{*}\left(X ; Z_{p}\right)$. Suppose there is a relation in $P(1) *(X)$ such that 


$$
w_{1} b_{1}+w_{2} b_{2}+\cdots+w_{s} b_{s}=0
$$

Then there is $y \in_{F(1)} E_{2}^{*, *}$ such that $d_{r_{t}}(y)=\lambda_{t} w_{t} i\left(b_{t}\right)$ in $\quad B P\left(p, J_{t-1}\right) E_{r_{t}}, 0 \neq \lambda_{t} \in Z$, for $1 \leqq t \leqq s$.

Proof. Using the argument similar to the proof of Lemma 2.1, we can construct a $\hat{J}_{s}$-manifold $\hat{y}$ such that

$$
\partial_{\jmath} y=w_{j} b_{\jmath}, \quad \text { i.e., } \quad \partial_{\jmath} \hat{y}=b_{\jmath} .
$$

Since $w_{\jmath} b_{\jmath}=0$ and $\delta_{\jmath} y=b_{\jmath}$ in $B P\left(p, J_{s}-\left\{w_{j}\right\}\right) *(X)$, it follows from Lemma 2.5 that

$$
d_{r_{j}}(y)=\lambda_{j} w_{j} b_{j} \text { in } B P\left(p, J_{s}-\left\{w_{j}\right\}\right) E_{r_{j}}^{* *} .
$$

That $B P\left(J_{j}\right)^{*} \cong B P\left(p, J_{s}-\left\{w_{j}\right\}\right)^{*}$ for $*<\left|w_{j+1}\right|$ implies the lemma. q.e.d.

For the preceding of this paper, we define an index which is convenient to use. If $x \in H^{*}\left(X ; Z_{p}\right)$ is in the image of $i: P(n)^{*}(X) \rightarrow H^{*}\left(X ; Z_{p}\right)$, then $x$ can be represented by a manifold with singularities of type $\left(p, v_{1}, \cdots, v_{n-1}\right)$.

Define $t(x)=n$ if $x$ is in Image $i: P(n)^{*}(X) \rightarrow H^{*}\left(X ; Z_{p}\right)$ and is not in Image $i: P(n-1) *(X) \rightarrow H^{*}\left(X ; Z_{p}\right)$.

From the facts that $Q_{\jmath}=i \delta_{j}, i r_{\alpha}=c\left(\mathcal{Q}^{\alpha}\right) i$, and $P(n) *(P(n)) \cong P(n)^{*} \bigotimes_{B P^{*}} B P^{*}(B P)$ $\otimes \Lambda\left[Q_{0}, \cdots, Q_{n-1}\right]$, we can easy see the following ;

$$
\begin{aligned}
& t(x)=n \quad \text { implies } \quad Q_{m} x=0 \text { for all } m \geqq n . \\
& Q_{n} x \neq 0 \quad \text { implies } \quad t(x) \geqq n+1 . \\
& t(x)=n \quad \text { implies } \quad t\left(Q_{n-1} x\right) \leqq n-1 . \\
& t\left(Q_{n} x\right) \leqq t(x) . \\
& t\left(\mathcal{P}^{\alpha} x\right) \leqq t(x) . \\
& t(x y) \leqq \max (t(x), t(y)) . \\
& \text { Given } f: X \rightarrow Y, \quad t\left(f^{*} x\right) \leqq t(x) .
\end{aligned}
$$

Question 2.7. Assume $t\left(b_{1}\right) \leqq 1\left(, t\left(b_{i}\right) \leqq 1\right)$ and there is a unique $z$ in $H^{*}\left(X ; Z_{p}\right)$ such that $Q_{1} z=b_{1}$ (, respectively $v_{k} b_{i}=0$ in $_{P(1)} E^{* *}$ for all $k<\imath$ and $Q_{\imath} z=b_{i}$ ). Then are there $b_{\jmath}^{\prime}$ in $B P^{*}\left(X ; Z_{p}\right)$ such that

$$
Q_{j} z=i\left(b_{\jmath}^{\prime}\right) \text { and } \Sigma v_{j} b_{\jmath}^{\prime}=0
$$

\section{§ 3. Examples}

3.1. The spectrum $V(n)$. 
THEOREM 3.1.1. (Larry Smith) Given a finite complex $X$, then $H^{*}\left(X ; Z_{p}\right)$ $\cong \Lambda\left[Q_{0}, \cdots, Q_{n}\right]$ if and only if $B P^{*}(X) \cong B P^{*} /\left(p, v_{1}, \cdots, v_{n}\right)$.

Proof. Assume $H^{*}\left(X ; Z_{p}\right) \cong \Delta\left[Q_{0}, \cdots, Q_{n}\right]$. Using Corollary 2.3, it is inductively proved that

$$
{ }_{P(1)}^{*} E_{2 p}^{*, *} \cong\left(\Lambda\left[Q_{0}, Q_{s+1}, \cdots, Q_{n}\right] \otimes B P^{*} /\left(p, v_{1}, \cdots, v_{s}\right)\right) Q_{1} \cdots Q_{s} .
$$

Hence we have $P(1)^{*}(X) \cong B P^{*} /\left(p, \cdots, v_{n}\right) \otimes \Lambda\left[Q_{0}\right]$ and $B P^{*}(X) \cong B P^{*} /\left(p, \cdots, v_{n}\right)$.

Conversely let $B P^{*}(X) \cong B P^{*} /\left(p, \cdots, v_{n}\right)$. From Lemma 2.1, there are $y_{r} \in$ $H^{*}\left(X ; Z_{p}\right)$ with $Q_{r} y_{r}=v_{r} x$ where $x$ is the $B P^{*}$-module generator of $B P^{*}(X)$. From Lemma $2.5, d_{s} y_{r}=v_{r} x$ in ${ }_{P(1)} E_{s}^{*, *}$. The $B P^{*}$-module generated by $y_{n}$ in $E_{2 p^{n-1}}$ is a $B P^{*} /\left(p, \cdots, v_{n-1}\right)$-free module, indeed, if $d_{2 p^{n-1}}$ is not monic then the $B P^{*}$-module generator of $\operatorname{kerd}_{2 p^{n-1}}$ is of the second degree $>-2 p^{n}+1$ and this contradicts to that the generator is not a permanent cycle. Hence we can take $y_{n, n-1}$ in $H^{*}\left(X ; Z_{p}\right)$ such that $Q_{n-1} y_{n, n-1}=y_{n}$.

Continuing this argument, there is $z$ such that $Q_{0} \cdots Q_{n} z=x$. Let $H^{*}\left(X ; Z_{p}\right)$ $\cong \Lambda\left[Q_{0}, \cdots, Q_{n}\right] z+B$. Each element in $B$ is not a permanent cycle in ${ }_{P(1)} E^{*, *}$. Let $w$ be a highest dimensional non zero element in $B$. Then $d w=v_{n} Q_{\imath_{1}} \cdots Q_{\imath_{s}} \bar{z}$ and this follows the contradiction. Therefore $B=0$.

Remark. Theorem 3.11 is also proved more easily by using the Sullivan exact sequence.

We show that all regular invariant ideals containing $p$ appear as annihilator ideals of some elements in $B P^{*}(X)$.

EXAMPLE 3.1.2. Let $J_{n}=\left(p, a_{1}, \cdots, a_{n}\right)$ be a regular invariant ideal of $B P^{*}$. Let $B P\left(J_{n}\right)$ be the spectrum of the bordism theory with the coefficient $B P^{*} / J_{n}$. The spectrum is inductively defined by the cofibering

$$
B P\left(J_{\imath}\right) \stackrel{a_{\imath+1}}{\longrightarrow} B P\left(J_{\imath}\right) \longrightarrow B P\left(J_{\imath+1}\right) .
$$

Using the fact $a_{\imath+1}^{*}=a_{\imath+1} \bmod J_{\imath}$, we can see (reference [9]) such as the case $J_{n}=I_{n+1}=\left(p, \cdots v_{n}\right)$

$$
\begin{aligned}
& B P^{*}\left(B P\left(J_{n}\right)\right) \cong B P^{*} / J_{n} \bigotimes_{B P^{*}} B P^{*}(B P) \text { and } \\
& P(M)^{*}\left(B P\left(J_{n}\right)\right) \cong P(M)^{*} \bigotimes_{B P^{*}} B P^{*}(B P) \otimes \Lambda\left[\bar{Q}_{0}, \cdots, \bar{Q}_{n}\right]
\end{aligned}
$$

for sufficient large $M$, e.g., $M>\left|a_{n}\right|$.

Let $B P\left(J_{n}\right)^{N}$ be an $N$-dimensional skeleton of $B P\left(J_{n}\right)$ (, note that $B P\left(J_{n}\right)^{N}$ is equivalent to a finite complex, because $\left.p \in J_{n}\right)$. The highest degree of the nonzero differential of the spectral sequence ${ }_{P(1)} E^{* * *}\left(B P\left(J_{n}\right)\right)$ is $\left|a_{n}\right|+1$. Hence we have

$$
B P^{*}\left(B P\left(J_{n}\right)^{N}\right) \cong B P^{*} / J_{n} \underset{B P^{*}}{\bigotimes} B P^{*}\left(B P^{N-\left|a_{n}\right|-2}\right) \oplus A
$$

where $A$ is the $B P^{*}$-module generated by generators $>N-\left|a_{n}\right|-2$. 
3.2. Lens space.

Let $X$ be a finite complex with $H^{\text {odd }}(X ; Z)=0$. Then the spectral sequence ${ }_{B P} E^{*} *(X)$ collapses.

THEOREM 3.2.1. Let $L$ be a $2 m+1$-dimensional generalized Lens space $L(p$, $\left.q_{1}, \cdots, q_{m}\right)$. Then there is a $B P^{*}$-algebra isomorphism

$$
B P *(L) \cong B P *[x] /\left(x^{m+1}, f(x)\right)
$$

where $f(x)=p x+a_{1} x^{2}+\cdots$, and $a_{p^{n-1}}=v_{n} \bmod \left(p, \cdots, v_{n-1}\right)$.

Proof. The cohomology ring is well known

$$
H *\left(L ; Z_{p}\right) \cong Z_{p}[x] /\left(x^{m+1}\right) \otimes \Lambda(\alpha), \quad Q_{0} \alpha=x .
$$

Since $H^{\text {odd }}(L ; Z)=0$, there is a $B P^{*}$-module isomorphism

$$
{ }_{B P} E_{\infty}^{*, *} \cong B P^{*}[x] /\left(p, x^{m+1}\right) .
$$

From Lemma 2.1 and $Q_{n} \alpha=x^{p^{n}}$, we have

$$
p x+a_{1} x^{2}+\cdots=f(x)=0 \text { in } B P^{*}(L) \text { and } a_{p_{n-1}}=v_{n} \bmod \left(p, \cdots, v_{n-1}\right) .
$$

q.e.d.

Remark. From the Gysin exact sequence, it is well known when $L=L$ $(p, 1, \cdots, 1)$, the polynomial $f(x)$ is the $p$-th product $[p]$ of the formal group law.

\subsection{Finite $H$-spaces}

Suppose that $W$ is a 1 -connected $(\bmod p)$ finite $H$-space. Let $Q$ be the $Z_{p^{-}}$ module of indecomposed elements in $H^{*}\left(W ; Z_{p}\right)$. The Kane's binary theorem [4] is stated as follows.

$$
\begin{aligned}
Q^{2 n}=\delta \mathscr{Q}^{P s-1} Q^{2 n-p^{s-1}}=Q_{s} Q^{2 n-p^{s}+1} & \text { for } s \geqq 0 \\
\text { if } Q^{2 n} \neq 0 \text { then } n=\left(p^{k+1}-1\right) /(p-1)-p^{l} & \text { for } 1 \leqq l \leqq k .
\end{aligned}
$$

Moreover for $a \in Q^{2 n}$

$$
\begin{aligned}
& a^{p}=0 \quad \text { if } \quad l=p^{k} \text { in (1), } \\
& a^{p^{2}=0} \text { otherwise. }
\end{aligned}
$$

Let denote by $\left(y_{k}, \cdots, y_{1}\right)$ the system of generators such that

$$
\begin{gathered}
\left|y_{l}\right|=\left(p^{k+1}-1\right) /(p-1)-p^{l} \\
\mathscr{P}^{p^{(l-1)}} y_{l}=y_{l-1} .
\end{gathered}
$$

Question 3.3.1. Is it true that $t\left(y_{k}\right)=0$ for all $k$ and

$$
v_{h} y_{l}^{\prime}+{ }_{\imath} v y_{h}^{\prime}=0 \bmod p \text { in } B P^{*}(W)
$$

where $i\left(y_{l}^{\prime}\right)=y_{l}, i\left(y_{h}^{\prime}\right)=y_{h}$ ? 
Remark 3.3.2. (1) Harper constructed [3] an $H$-space for each odd prime $p$ such as

$$
H^{*}\left(W ; Z_{p}\right)=\wedge\left(x_{3}, x_{2 p+1}\right) \otimes Z_{p}\left[x_{2 p+2}\right] /\left(x_{2 p+2}^{p}\right) .
$$

Then by the arguments similar to [10],

$$
\begin{aligned}
B P^{*}(W) \cong B P^{*}\left\{1, y_{3}, y_{a}\right\} & \oplus B P^{*}\left\{y_{b}, y_{c}\right\} /\left(p y_{b}=v_{1} y_{c}\right) \\
& \oplus B P^{*} /\left(p, v_{1}\right)\left[x_{2 p+2}\right] /\left(x_{2 p+2}\right)^{p},
\end{aligned}
$$

where $a=2 p^{2}+2 p+2, b=a-3, c=2 p^{2}+1$.

(2) The cohomology ring of the exceptional Lid group $E_{8}$ for $p=3$ is

$$
H^{*}\left(E_{8} ; Z_{3}\right) \cong Z_{3}\left[x_{8}, x_{20}\right] /\left(x_{8}^{3}, x_{20}^{3}\right) \otimes \Lambda
$$

where $\Lambda$ is the external product of odd dimensional generators. The $B P^{*}$. module structure of $B P^{*}\left(E_{8}\right)$ is known [11]. It holds that $t\left(x_{8}\right)=0$ and hence $v_{1} x_{8}=v_{2} x_{20}$.

It is unknown whether there exists an $H$-space such that $k \geqq 3$ in the binary theorem (2).

3.4. Eilenberg-MacLane space $K(Z, 3)$.

The mod $\mathrm{p}$ cohomology of $K(Z, 3)$ is known

$$
H^{*}\left(K(Z, 3) ; Z_{p}\right) \cong Z_{p}\left[\delta \mathscr{P} \tau, \delta \mathscr{P}^{P} \mathscr{Q} \tau, \cdots\right] \otimes \Lambda[\tau, \mathscr{P} \tau, \cdots] .
$$

For simplicity of notations, let denote $\mathscr{Q}^{P^{n-1}} \cdots \mathscr{Q} \tau=c_{n}, \delta c_{n}=b_{n}$. Then $\left|c_{n}\right|=$ $2\left(p^{n}-1\right)+3,\left|b_{n}\right|=2\left(p^{n}-1\right)+4$.

Lemma 3.4.1. In $H^{*}\left(K(Z, 3) ; Z_{p}\right)$, the Milnor operations act

$\begin{array}{lll}\text { (1) } Q_{m} \tau=b_{m} & \text { (2) } Q_{m} b_{n}=0\end{array}$

(3) $Q_{m} c_{n}=Q_{n} c_{m}=\left(b_{n-m}\right)^{p^{m}}$ for $n>m>0$ and $Q_{m} c_{m}=0$.

Proof. The cohomology ring $H^{*}\left(K ; Z_{p}\right)$ is a Hopf algebra and, $c_{n}, b_{n}$ are primitive elements. By the definition, we have (1) and $Q_{0} c_{n}=b_{n}$. We show $Q_{m}\left(\mathscr{Q}^{P^{n-1}} c_{n-1}\right)=\left(Q_{m-1} c_{n-1}\right)^{p}$, indeed,

$$
\begin{aligned}
Q_{m}\left(\mathscr{P}^{P^{n-1}} c_{n-1}\right) & =\mathscr{Q}^{P^{n-1}+P^{m-1}} Q_{m-1} c_{n-1}-Q_{m-1} \mathscr{P}^{P^{n-1}+P^{m-1}} c_{n-1} \\
& =\left(Q_{m-1} c_{m-1}\right)^{p}\left(\text {, since }\left|Q_{m-1} c_{m-1}\right|=2\left(p^{n-1}+p^{m-1}\right)\right) .
\end{aligned}
$$

Hence inductively we have $Q_{m} c_{n}=\left(b_{n-m}^{P m-1}\right)^{p}$.

Since $Q_{m}$ is a derivation, $Q_{m}\left(b_{n}\right)$ is also primitive. Hence $Q_{m}\left(b_{n}\right)$ is an indecomposed element or its $p$-th power. By dimensional reason, we have (2).

q.e.d.

Theorem 3.4.2. There exist $b_{\jmath}^{\prime} \in B P^{*}(K(Z, 3))$ such that $i\left(b_{\jmath}^{\prime}\right)=b_{\jmath}$ and $v_{1} b_{1}^{\prime}+$ $v_{2} b_{2}^{\prime}+\cdots=0$. 
To prove this theorem we recall the Wilson's theorem. Let $B P\langle n\rangle=B P$ $\left(v_{n+1}, \cdots\right)$, namely, $B P\langle n\rangle_{*}=Z_{(p)}\left[v_{1}, \cdots, v_{n}\right]$.

THEOREM 3.4.3. (Wilson [13]) For $k \leqq 2\left(p^{n}+\cdots+p+1\right)$,

$$
i: B P^{k}(X) \longrightarrow B P\langle n\rangle^{k}(X) \quad \text { is epic. }
$$

Proof of Theorem 3.4.2. Since $b_{1}=Q_{0} c_{1}=Q_{1} \tau$,

$$
b_{1} \in \text { Image }\left(B P\langle 1\rangle *(K(Z, 3)) \longrightarrow H^{*}\left(K(Z, 3), Z_{p}\right)\right) .
$$

Moreover if $i\left(b_{1}^{\prime \prime}\right)=b_{1}$ then $v_{1} b_{1}^{\prime \prime}=0$. By Wilson's theorem, $\left|b_{1}^{\prime \prime}\right|=2(p+1)$ implies

$$
b_{1}^{\prime \prime} \in \operatorname{Image}\left(B P^{*}(K(Z, 3)) \longrightarrow B P\langle 1\rangle^{*}(K(Z, 3))\right. \text {. }
$$

Therefore $t\left(b_{1}\right)=0$ and let $i\left(b_{1}^{\prime}\right)=b_{1}$.

From Sullivan's exact sequence, there is $b_{2}^{\prime \prime}$ such that

$$
v_{1} b_{1}=-v_{2} b_{2}^{\prime \prime} \quad \text { in } B P\langle 2\rangle *(X) .
$$

Moreover from Lemma 2.1, $i\left(b_{2}^{\prime \prime}\right)=b_{2}$. By also Wilson's theorem, $\left|b_{2}^{\prime \prime}\right|<2\left(p^{2}+p+1\right)$ implies

$$
b_{2}^{\prime \prime} \in \operatorname{Image}\left(B P^{*}(K(Z, 3)) \longrightarrow B P\langle 2\rangle^{*}(X)\right) .
$$

Take $b_{2}^{\prime}$ such as $i\left(b_{2}^{\prime}\right)=b_{2}^{\prime \prime}$. Continuing this argument. We have the theorem.

q.e.d.

THEOREM 3.4.4. Let the filtration $F_{S}=\operatorname{Ker}\left(P(1) *(X) \rightarrow P(1)^{*}\left(X^{S}\right)\right)$. Then there is a $P(1)^{*}$-module isomorphism

$$
P(1) *(K(Z, 3)) / F_{2 p^{3+2}} \cong P(1) *\left[b_{1}, b_{2}, b_{3}\right] /(R, D)
$$

where $D$ is the ideal of elements of degree $\geqq 2 p^{3}+2$ in $Z_{p}\left[b_{1}, b_{2}, b_{3}\right]$ and $R$ is the ideal generated by the following five relations

$$
\begin{aligned}
& v_{1} b_{1}+v_{2} b_{2}+v_{3} b_{3}=0 \bmod I_{3}^{2} \\
& v_{1}^{p} b_{2}+v_{2} b_{1}^{p}+v_{3} b_{2}^{p}=0 \bmod I_{3}^{2}-\left\{v_{1}\right\}^{2} \\
& v_{2} b_{1}^{p+1}+v_{3} b_{2}^{p} b_{1}=0 \bmod I_{3}^{2} \\
& v_{2} b_{1}^{p-1} b_{2}+v_{3}\left(b_{1}^{p^{2}}-b_{1}^{p-1} b_{3}\right)=0 \bmod I_{3}^{2} \\
& v_{1}^{p^{2}+1} b_{3}=0 \bmod I_{3}^{2}-\left\{v_{1}^{2}\right\} .
\end{aligned}
$$

Proof. We compute the Atiyah-Hirzebruch spectral sequence

$$
E_{2}=H^{*}\left(K(Z, 3), P(1)^{*}\right) \Rightarrow P(1)^{*}(K(Z, 3)) .
$$

The first non zero differential is $d_{2 p-1}=v_{1} \otimes Q_{1}$ and $Q_{1}$ acts such as 


$$
\tau \longrightarrow b_{1}, \quad c_{1} \longrightarrow 0, \quad c_{2} \longrightarrow b_{1}^{p}, \quad c_{3} \longrightarrow b_{2}^{p}, \cdots
$$

Hence we have

$$
E_{2 p}^{s, *} \cong P(1) *\left[b_{1}, \cdots\right] \otimes \Lambda\left(c_{1}, c_{2}-b_{1}^{p-1} \tau\right) /\left(v_{1} b_{1}^{p}, v_{1} b_{1}^{p}, \cdots\right) .
$$

Each element $x$ of $\left|c_{1}\right|<|x|<\left|b_{2}\right|$ or $\left|c_{1}\right|+\left|c_{2}\right|<|x|<\left|b_{2}^{p}\right|$ is $v_{1}$-torsion in $E_{2 p}$. Since $K(1)^{*}(K(Z),) \cong 0, v_{1}^{s} b_{2} \in \operatorname{Imd}_{r}$ and we have

$$
d_{2(p-1) p+1} c_{1}=v_{1}^{p} b_{2} .
$$

Since $d_{s}\left(c_{2}-b_{1}^{p-1} \tau\right)=0$ for $s \leqq 2(p-1) p+1$, we also have

$$
d_{2(p-1) p+1} c_{1}\left(c_{2}-b_{1}^{p-1} \tau\right)=v_{1}^{p} b_{2}\left(c_{2}-b_{1}^{p-1}\right) \text {. }
$$

Hence if $s \leqq 2 p^{3}+2$, then

$$
E_{2\left(p^{2-1}\right)}^{s, *} \cong P(1)\left[b_{1}, b_{2}, b_{3}\right] \otimes \wedge\left(c_{1} b_{1}, c_{2} b_{1}^{p-1}\right) /\left(v_{1} c_{1} b_{1}, v_{1} b_{1}^{p}, v_{1}^{p} b_{2}\right) .
$$

It is easily seen $v_{1}^{p} x=0$ for $\left|c_{1}\right|+\left|c_{2}\right|<|x|<\left|v_{2}\right|+\left|c_{2}\right|$. The next non zero differential is $d_{2 p^{2+1}}=v_{2} \otimes Q_{2}$. The operation $Q_{2}$ acts

(i) $c_{1} b_{1} \rightarrow v_{2} b_{1}^{p+1}$; both sides are $P(1)^{*} / v_{1}$-free,

(ii) $c_{2}-b_{1}^{p-1} \tau \rightarrow-v_{2} b_{1}^{p-1} b_{2}$; the left side is $P(1)^{*}$-free and the other is $v_{1^{-}}$torsion,

(iii) $\quad c_{1}\left(c_{2}-b_{1}^{p-1} \tau\right) \rightarrow v_{2}\left(b_{1}^{p}\left(c_{2}-b_{1}^{p-1} \tau\right)+c_{1} b_{1}^{p-1} b_{2}\right)$; both sides are $P(1) / v_{1}$-free.

Therefore if $s \leqq 2 p^{3}+2$,

$$
\begin{aligned}
E_{2 p^{2}}^{s, *} \cong & P(1) *\left[b_{1}, b_{2}, b_{3}\right] \otimes\left\{1, v_{1} \otimes\left(c_{2}-b_{1}^{p-1} \tau\right), b_{1}^{p}\left(c_{2}-b_{1}^{p-1} \tau\right)+c_{1} b_{1}^{p-1} b_{2}\right\} / \\
& \left(v_{1} b_{1}, v_{1}^{p} b_{2}, v_{2} b_{1}^{p+1}, v_{2} b_{1}^{p-1} b_{2},\left(v_{1}, v_{2}\right)\left(b_{1}^{p}\left(c_{2}-b_{1}^{p-1} \tau\right)+c_{1} b_{1}^{p-1} b_{2}\right)\right)
\end{aligned}
$$

We will see odd dimensional elements are not permanent. Since $K(1)^{*}$ $(K(Z, 3)) \cong 0, v_{1}^{s} b_{3} \in \operatorname{Imd}_{r}$. The $P(1)^{*}$-free generator of dimension $<\left|b_{3}\right|$ is only one and

$$
d_{r}\left(v_{1} \otimes c_{2}-b_{1}^{p-1} \tau\right)=v_{1}^{p^{2}+1} b_{3} .
$$

Since $d_{2 p^{3+1}}=v_{3} \otimes Q_{3} \bmod \left(v_{1}, v_{2}\right)$, we have

$$
d_{2 p^{3+1}}\left(b_{1}^{p}\left(c_{2}-b_{1}^{p-1} \tau\right)+c_{1} b_{1}^{p-1} b_{2}\right)=v_{3}\left(b_{1}^{p+p^{2}}-b_{1}^{2 p-1} b_{3}+b_{2}^{p+1} b_{1}^{p+1}\right) .
$$

Hence for $s \leqq 2 p^{3}+2$

$$
E_{2 p^{2}}^{s_{1}} \cong P(1) *\left[b_{1}, b_{2}, b_{3}\right] /\left(v_{1} b_{1}, v_{1}^{p} b_{2}, v_{2} b_{1}^{p+1}, v_{2} b_{1}^{p-1} b_{2}, v_{1}^{p^{2}+1} b_{3}\right) .
$$

By Lemma 2.1 and Lemma 2.2, we have the relation, for example, the derivations $d_{r} c_{1}=v_{1}^{p} b_{2}, Q_{2} c_{1}=b_{1}^{p}, Q_{3} c_{1}=b_{2}^{p}$ imply relation (2).

q.e.d. 


\section{REFERENCES}

[1] N. BAAs, On bordism theories of manifolds with singularities, Math. Scand., 33 (1973), 279-302.

$52]$ W. Browder, On differential Hopf algebras, Trans. Amer. Math. Soc., 107 (1963), 153-176.

[3] J. Harper, On the construction of $\bmod p H$-spaces, Proc. Symp. Pure Math., 32 (1978), 207-214.

[4] R. Kane, BP-torsion in finite $H$-spaces, Trans. AMS 264 (1981), 473-497.

5. D. Quillen, Elementary proofs of some results of cobordism theory using Steenrod operations, Advanced Math., 7 (1971) 29-56.

[6] L. Smith, On realizing complex cobordism modules, Amer. J. Math. 92 (1970), 793-856.

[7] D. Sullivan, Geometric seminor notes, Princeton Univ., (1967).

[8] H. Toda, On realizing exterior parts of the Steenrod algebras, Topology 10 (1971), 53-65.

[9] N. YaGita, On the algebraic structure of cobordism operation with singularities, J. London Math. Soc. 16 (1977), 131-141.

[10] N. Yagita, Brown-Peterson cohomology groups of exceptional Lie groups, J. Pure and Appplied Algebra 17 (1980), 223-226.

$\left[117 \mathrm{~N}\right.$. Yagita, The $B P^{*}$-module structure of $B P^{*}\left(E_{8}\right)$ for $p=3$, preprint.

[12] D. Ravenel and S. Wilson, The Morava $K$-theories of Eilenberg-MacLane spaces and the Conner-Floyd conjecture, Amer. J. Math., 102 (1980), 691-748.

[13] S. Wilson, The $\Omega$-spectrum for Brown-Peterson cohomology II Amer. J. Math., 97 (1975), 101-123.

Department of Mathematics

Musashi Institute of Technology

Tamazutsumi Setagaya Tokyo Japan 
